\title{
Comparative prostate MRI before and after chronic granulomatous prostatitis following intravesical bacillus Calmette-Guérin therapy
}

Julien Sarkis*,1(i), Georges Nawfal², Elias El-Haddad ${ }^{3}$ (iD), Georges Abi Tayeh ${ }^{1}$ (i), Nathalie Mahfoud $^{4} \&$ Pierre Sarkis 5

${ }^{1}$ Department of Urology, Hôtel-Dieu de France Hospital, Beirut, Lebanon

${ }^{2}$ Department of Radiology, Saint Joseph Hospital, Beirut, Lebanon

${ }^{3}$ Department of Radiology, Hôtel-Dieu de France Hospital, Beirut, Lebanon

${ }^{4}$ Department of Pathology, Saint Joseph Hospital, Beirut, Lebanon

${ }^{5}$ Department of Urology, Saint Joseph Hospital, Beirut, Lebanon

*Author for correspondence: Tel.: +961 7063 2826; juliensarkis@live.com

Background: Granulomatous prostatitis $(\mathrm{GnP})$ is an interesting complication of bacillus Calmette-Guérin (BCG) therapy as it mimics prostate cancer on clinical, biochemical and imaging examinations. In the era of multiparametric prostate MRI (mpMRI), differentiation of GnP from prostate cancer on imaging is essential. Case presentation: We report a case of post-BCG GnP in a patient with nonmuscle invasive bladder cancer, presenting with a prostate-specific antigen level of $21.6 \mathrm{ng} / \mathrm{ml}$ and prostate imaging reporting and data system (PI-RADS) 5 peripheral lesions. A mpMRI performed 6 months before showed a score 2 of PI-RADS. Conclusion: The comparison of mpMRI images before and after BCG administration gives urologists, oncologists and radiologists a precise idea of the mpMRI changes that occur following BCG administration to eventually prevent unnecessary biopsies in future patients.

Lay abstract: Granulomatous prostatitis is a bacillus Calmette-Guérin therapy complication that often mimics prostate cancer. This article gives radiologists, urologists and oncologists a clear multiparametric prostate MRI comparison of the changes that bacillus Calmette-Guérin prostatitis causes on the prostate gland.

First draft submitted: 11 April 2020; Accepted for publication: 16 September 2020; Published online: 20 October 2020

Keywords: BCG therapy $\bullet$ granulomatous prostatitis $\bullet$ prostate cancer $\bullet$ prostate MRI $\bullet$ prostate-specific antigen $\bullet$ PSA

Bacillus Calmette-Guérin (BCG) is recommended as intravesical immunotherapy in intermediate- and high-risk nonmuscle invasive bladder cancer [1]. Granulomatous prostatitis $(\mathrm{GnP})$ is an interesting complication of BCG therapy, accounting for approximatively $1.3 \%$ of patients [2]. It mimics prostate cancer on clinical, biochemical and imaging examinations [3]. Despite the recent advances in prostate imaging, the MRI appearance of GnP after BCG therapy is not well described due to its relative rarity [4].

We herein present a case of GnP diagnosed 2 months after completion of a 6-week course of BCG, on the basis of high prostate-specific antigen (PSA) and score 5 of prostate imaging reporting and data system (PI-RADS) on multiparametric prostate MRI (mpMRI). Of note, a previous mpMRI performed 6 months before showed a score 2 of PI-RADS.

\section{Case presentation}

A 72-year-old male patient presented for elevated PSA of $6.5 \mathrm{ng} / \mathrm{ml}$ (normal PSA $<4.0 \mathrm{ng} / \mathrm{ml}$ ) with a normal digital rectal exam. A mpMRI performed showed benign (PI-RADS 2) lesions at the peripheral zone (Figure 1). Consequently, prostate biopsies were deemed unnecessary and a PSA follow-up decided after 6 months. 


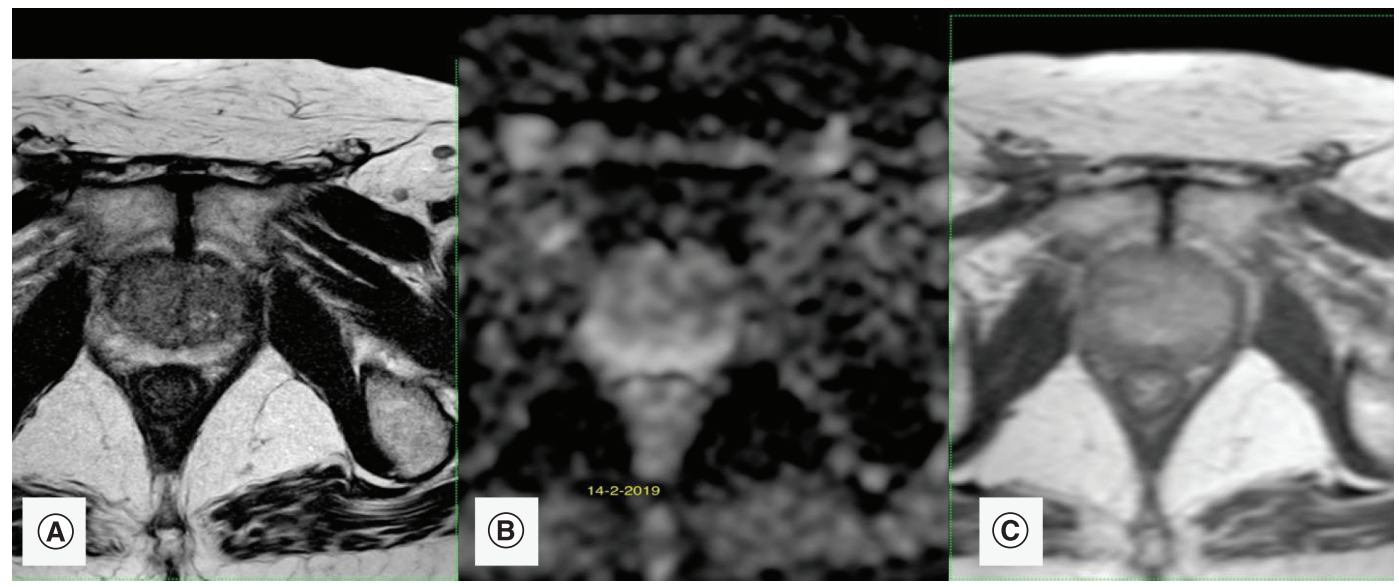

Figure 1. Multiparametric prostatic MRI before intravesical bacillus Calmette-Guérin therapy. (A) Axial T2-weighted image shows peripheral symmetrical hypointense linear lesions corresponding to sequelae of chronic

prostatitis. (B) Apparent diffusion coefficient map of the diffusion-weighted image shows no corresponding diffusion restriction in peripheral zones. (C) Dynamic contrast enhancement shows only mild enhancement mainly in the left peripheral zone. These findings suggest sequelae of chronic prostatitis and a PI-RADS score of 2.

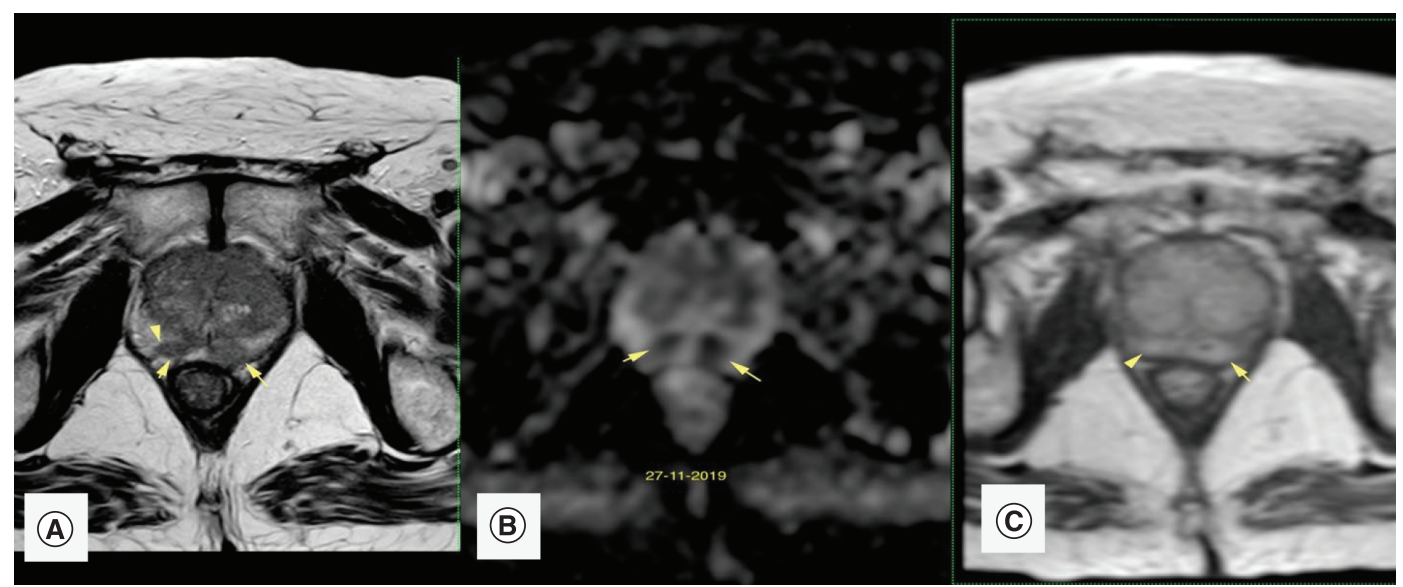

Figure 2. Multiparametric prostatic MRI after intravesical bacillus Calmette-Guérin therapy. (A) Axial T2-weighted image: a focal and ill-defined hypointense lesion in the right and left PZ (arrow) exhibits a bulging contour (arrowhead), suspicious of prostate cancer. (B) Apparent diffusion coefficient map: the PZ lesions (arrows) exhibit very low signal intensity in the apparent diffusion coefficient map. This is compatible with significant diffusion restriction in those areas. (C) Dynamic contrast enhancement shows moderate to significant early and diffuse enhancement mainly in the PZ. These findings are compatible with a PI-RADS score of 5.

PZ: Peripheral zone.

A month later he developed isolated macroscopic hematuria with work-up revealing an intermediate-risk nonmuscle invasive bladder cancer (multifocal low grade bladder tumor pTa LG) managed with a complete transurethral resection of the bladder and subsequent 6-week course of intravesical BCG therapy.

During his follow-up 2 months after his last BCG instillation, PSA level rose to $21.6 \mathrm{ng} / \mathrm{ml}$, with a repeated dosage of $22 \mathrm{ng} / \mathrm{ml}$ after 2 weeks. The patient was asymptomatic. Urine culture was negative and rectal examination was not suspicious. A new mpMRI showed multiple PI-RADS 5 lesions in the right and left peripheral zone (Figure 2). Despite the clinical suspicion of granulomatous prostatitis, patient anxiety as well as high PSA levels pushed us toward performing prostate biopsies. MRI guided in-bore prostate biopsies of the lesions were realized, showing an inflammatory process with noncaseating granulomatous reaction with no sign of malignancy (Figure 3), all in favor of granulomatous prostatitis secondary to intravesical BCG therapy. No antituberculous treatment was started. Patient was followed up with a PSA of $14.4 \mathrm{ng} / \mathrm{ml}$ after 3 months. 

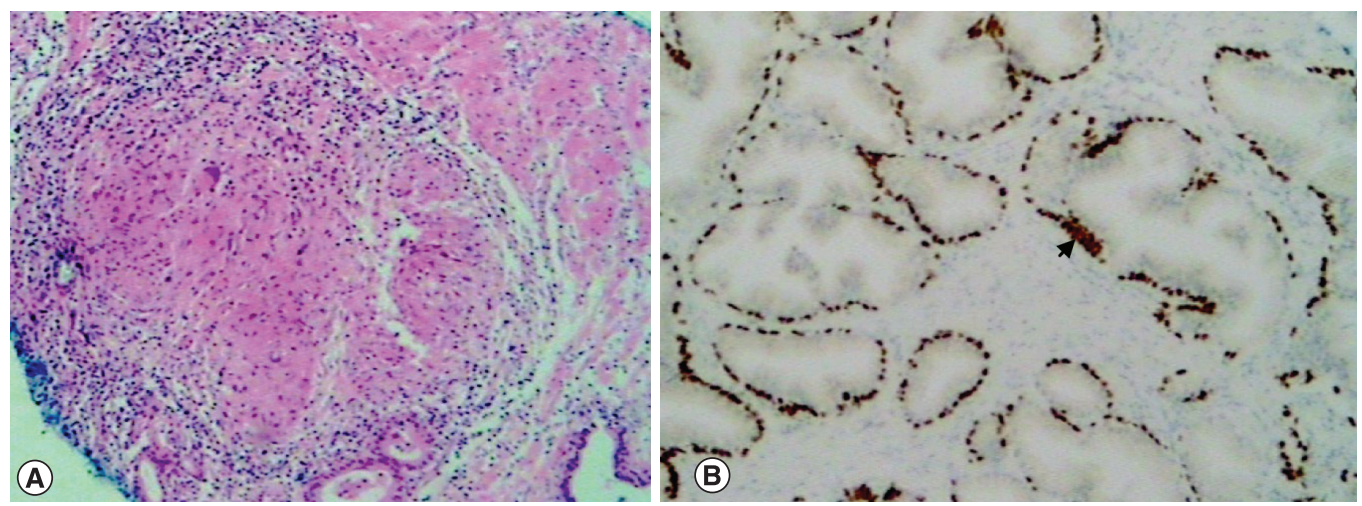

Figure 3. Histopathological findings of in-bore prostate biopsy. (A) Hematoxylin and eosin staining showing a non-necrotizing granuloma composed of epithelioid histiocytes and giant cells. (B) Immunochemistry highlighting normal prostatic glands with positive p63 basal cells (arrowhead), not in favor of prostate cancer.

\section{Discussion}

Intravesical instillation of BCG is recommended following transurethral resection of the bladder in the setting of intermediate- and high-risk nonmuscle invasive bladder cancer, reducing cancer progression and recurrence [1]. Although rare, adverse effects of BCG therapy can range from local side effects like BCG cystitis, to more serious systemic BCG infections [5]. In addition, over $40 \%$ of patients present a significant increase in their PSA level [6,7] with prostatic granulomas often mimicking prostatic neoplasm on clinical [8] and imaging examinations (accounting to over $6 \%$ of PI-RADS-5 lesions on mpMRI [9]).

Few reports describe the MRI features of $\mathrm{GnP}$, which are nonspecific. In a retrospective case series of ten patients, Suzuki et al. concluded that GnP generally appears hypointense relative to the surrounding peripheral zone on T2-weighted images and hyperintense on diffusion-weighted images [10], without mentioning the corresponding PI-RADS score. This appearance is variable depending on the amount of free radicals produced by the inflammatory process $[4,10]$. With dynamic contrast enhancement, prostatic granulomas do not exhibit the rapid wash-in and wash-out seen in prostate cancer. However, indeterminate abnormalities would almost certainly require biopsy regardless of how well the imaging features are analyzed, especially in those with elevated PSA [11].

What is interesting in our patient is that a previous mpMRI had been done 4 months ago before BCG therapy showing a basically normal to mild prostatic abnormalities (score 2), with prominent nodular markedly hypointense diffuse lesions appearing on the latter MRI done after BCG therapy (score 5). Comparing both mpMRI can give urologists and radiologists a more precise idea of the imaging changes following BCG administration to eventually prevent unnecessary biopsies in future patients.

In addition, in a prospective study by Leibovici et al., PSA levels rose to a maximal average of $6.97 \mathrm{ng} / \mathrm{ml}$ during BCG treatment [4]. Our patient presented a PSA elevation to $22 \mathrm{ng} / \mathrm{ml}$, higher than the values reported in the literature pushing us toward performing prostate biopsies.

In this case, we only performed a targeted prostate biopsy. This could be debatable as extended plus targeted biopsy are strongly recommended at first presentation. However, our patient had a focal PI-RADS-5 lesion on mpMRI and the diagnosis of granulomatous prostatitis was highly probable considering the chronology of events. In their study, Grey et al. found that PI-RADS-5 lesions had specificity of 96.5\% (95\% confidence interval [CI] 91.7-98.7) for prostate cancer [12]. Therefore, to avoid the number of biopsy scores and to lower the risk of prostate biopsy complications, only targeted biopsies were performed.

No clear guidelines exist concerning the correct treatment of granulomatous prostatitis. Since our patient had no urinary or systemic symptoms and because of the absence of caseous necrosis on histology, he was managed conservatively with PSA follow-up and without antituberculosis treatment [13].

\section{Conclusion \& future perspective}

$\mathrm{GnP}$ is a known complication of intravesical BCG. When it is associated with a high PSA and a suspicious clinical exam, it can easily be confounded with prostate cancer. The current report has the advantage of exposing the exact 
mpMRI changes following BCG, as the patient had an MRI before and after BCG administration. Additional comparative MRI analysis are of course needed, to reduce the number of unnecessary biopsies in GnP patients.

\section{Executive summary}

- Granulomatous prostatitis $(\mathrm{GnP})$ is an interesting complication of $\mathrm{BCG}$ therapy that mimics prostate cancer on clinical, biochemical and imaging examinations.

- We present a case of GnP diagnosed 2 months after completion of a 6-week course of BCG, on the basis of high prostate-specific antigen and score 5 of PI-RADS on multiparametric prostate MRI (mpMRI). Of note, a previous mpMRI performed 6 months before showed a score 2 of PI-RADS.

- Few reports describe the MRI characteristics of GnP, which are nonspecific. Our case describes the exact features of GnP on mpMRI.

- No clear guidelines exist concerning the correct treatment of GnP. Our patient had an asymptomatic GnP. The patient was managed conservatively without antituberculosis therapy, with a decrease of his prostate-specific antigen after 6 months.

Author contributions

J Sarkis contributed to the conception of the case report, data collection and drafting and critical revision of the article. E El-Haddad and G Abi Tayeh contributed to collection and assembly of data and revision of the article. N Mahfoud made contributions in drafting the article. P Sarkis and G Nawfal contributed to data collection and revision of the article. P Hajj contributed to conception of the case report and final approval of the article.

\section{Acknowledgments}

The work has been reported in line with the CARE guidelines for case reporting.

Financial \& competing interests disclosure

The authors have no relevant affiliations or financial involvement with any organization or entity with a financial interest in or financial conflict with the subject matter or materials discussed in the manuscript. This includes employment, consultancies, honoraria, stock ownership or options, expert testimony, grants or patents received or pending, or royalties.

No writing assistance was utilized in the production of this manuscript.

\section{Informed consent disclosure}

The authors state that they have obtained appropriate institutional review board approval or have followed the principles outlined in the Declaration of Helsinki for all human or animal experimental investigations. The authors state that they have obtained verbal and written informed consent from the patient for the inclusion of their medical and treatment history within this case report.

\section{Open access}

This work is licensed under the Creative Commons Attribution 4.0 License. To view a copy of this license, visit http://creativecomm ons.org/licenses/by/4.0/

\section{References}

1. Babjuk M, Burger M, Compérat EM et al. European Association of Urology guidelines on nonmuscle-invasive bladder cancer (TaT1 and carcinoma in situ) - 2019 update. Eur. Urol. 76(5), 639-657 (2019).

2. Lamm DL, van der Meijden PM, Morales A et al. Incidence and treatment of complications of bacillus Calmette-Guérin intravesical therapy in superficial bladder cancer. J. Urol. 147(3), 596-600 (1992).

3. Uzoh CC, Uff JS, Okeke AA. Granulomatous prostatitis. BJU Int. 99(3), 510-512 (2007).

4. Diaz de Leon A, Costa DN, Francis F, Pedrosa I. Case 258: granulomatous prostatitis. Radiology 289(1), 267-271 (2018).

5. Decaestecker K, Oosterlinck W. Managing the adverse events of intravesical bacillus Calmette-Guérin therapy. Res. Rep. Urol. 7 , 157-163 (2015).

6. Leibovici D, Zisman A, Chen-levyi Z et al. Elevated prostate specific antigen serum levels after intravesical instillation of bacillus Calmette-Guerin. J. Urol. 164(5), 1546-1549 (2000).

7. Beltrami P, Ruggera L, Cazzoletti L, Schiavone D, Zattoni F. Are prostate biopsies mandatory in patients with prostate-specific antigen increase during intravesical immuno- or chemotherapy for superficial bladder cancer? Prostate 68(11), 1241-1247 (2008). 
8. Okçelik S, Soydan H, Yılmaz İ, Yılmaz Ö, Ateş F, Karademir K. Granulomatous prostatitis diagnosed during intravesical BCG treatment. Turk. J. Urol. 39(3), 204-206 (2013).

9. Pepe P, Pennisi M. Negative biopsy histology in men with PI-RADS score 5 in daily clinical practice: incidence of granulomatous prostatitis. Clin. Genitourin. Cancer doi:10.1016/j.clgc.2020.04.001 (2020) (Epub ahead of print).

10. Suzuki T, Takeuchi M, Naiki T et al. MRI findings of granulomatous prostatitis developing after intravesical bacillus Calmette-Guérin therapy. Clin. Radiol. 68(6), 595-599 (2013).

11. Ma W, Kang SK, Hricak H, Gerst SR, Zhang J. Imaging appearance of granulomatous disease after intravesical bacille Calmette-Guérin (BCG) treatment of bladder carcinoma. Am. J. Roentgenol. 192(6), 1494-1500 (2009).

12. Grey ADR, Chana MS, Popert R, Wolfe K, Liyanage SH, Acher PL. Diagnostic accuracy of magnetic resonance imaging (MRI) prostate imaging reporting and data system (PI-RADS) scoring in a transperineal prostate biopsy setting. BJU Int. 115(5), 728-735 (2015).

13. Lee SY, Choi SH. Treatment experience for incidentally diagnosed asymptomatic prostate tuberculosis in a patient with history of BCG intravesical therapy. Urol. Case Rep. 17, 39-41 (2018). 